\title{
Natriuretic Peptide-Guided Therapy in Chronic Heart Failure: A Meta-Analysis of 2,686 Patients in 12 Randomized Trials
}

\author{
Gianluigi Savarese ${ }^{1}$, Bruno Trimarco ${ }^{1}$, Santo Dellegrottaglie ${ }^{1,2}$, Maria Prastaro ${ }^{1}$, \\ Francesco Gambardella ${ }^{1}$, Giuseppe Rengo ${ }^{3}$, Dario Leosco ${ }^{3}$, Pasquale Perrone-Filardi ${ }^{1}$ *
}

1 Department of Advanced Biomedical Sciences, Federico II University, Naples, Italy, 2 Division of Cardiology, Ospedale Medico-Chirurgico Accreditato Villa dei Fiori, Acerra, Naples, Italy, 3 Department of Medical Translational Sciences, Federico II University, Naples, Italy

\begin{abstract}
Background: The role of cardiac natriuretic peptides in the management of patients with chronic heart failure (HF) remains uncertain. The purpose of this study was to evaluate whether natriuretic peptide-guided therapy, compared to clinicallyguided therapy, improves mortality and hospitalization rate in patients with chronic HF.

Methodology/Principal Findings: MEDLINE, Cochrane, ISI Web of Science and SCOPUS databases were searched for articles reporting natriuretic peptide-guided therapy in HF until August 2012. All randomized trials reporting clinical end-points (allcause mortality and/or HF-related hospitalization and/or all-cause hospitalization) were included. Meta-analysis was performed to assess the influence of treatment on outcomes. Sensitivity analysis was performed to test the influence of potential effect modifiers and of each trial included in meta-analysis on results. Twelve trials enrolling 2,686 participants were included. Natriuretic peptide-guided therapy (either B-type natriuretic peptide [BNP]- or N-terminal pro-B-type natriuretic peptide [NT-proBNP]-guided therapy) significantly reduced all-cause mortality (Odds Ratio [OR]:0.738; 95\% Confidence Interval [CI]:0.596 to 0.913; $\mathrm{p}=0.005$ ) and HF-related hospitalization (OR:0.554; $\mathrm{Cl}: 0.399$ to $0.769 ; \mathrm{p}=0.000$ ), but not all-cause hospitalization (OR:0.803; $\mathrm{Cl}: 0.629$ to $1.024 ; \mathrm{p}=0.077$ ). When separately assessed, NT-proBNP-guided therapy significantly reduced all-cause mortality (OR:0.717; $\mathrm{Cl}: 0.563$ to $0.914 ; \mathrm{p}=0.007$ ) and HF-related hospitalization (OR:0.531; $\mathrm{Cl}: 0.347$ to $0.811 ; \mathrm{p}=0.003$ ), but not all-cause hospitalization (OR:0.779; $\mathrm{Cl}: 0.414$ to $1.465 ; \mathrm{p}=0.438$ ), whereas BNP-guided therapy did not significantly reduce all-cause mortality (OR:0.814; $\mathrm{Cl}: 0.518$ to $1.279 ; \mathrm{p}=0.371$ ), HF-related hospitalization (OR:0.599; $\mathrm{Cl}: 0.303$ to $1.187 ; \mathrm{p}=0.142$ ) or all-cause hospitalization (OR:0.726; Cl:0.609 to $0.964 ; \mathrm{p}=0.077$ ).
\end{abstract}

Conclusions/Significance: Use of cardiac peptides to guide pharmacologic therapy significantly reduces mortality and HF related hospitalization in patients with chronic HF. In particular, NT-proBNP-guided therapy reduced all-cause mortality and HF-related hospitalization but not all-cause hospitalization, whereas BNP-guided therapy did not significantly reduce both mortality and morbidity.

Citation: Savarese G, Trimarco B, Dellegrottaglie S, Prastaro M, Gambardella F, et al. (2013) Natriuretic Peptide-Guided Therapy in Chronic Heart Failure: A MetaAnalysis of 2,686 Patients in 12 Randomized Trials. PLoS ONE 8(3): e58287. doi:10.1371/journal.pone.0058287

Editor: Adrian V. Hernandez, Universidad Peruana de Ciencias Aplicadas (UPC), Peru

Received January 3, 2013; Accepted February 1, 2013; Published March 5, 2013

Copyright: () 2013 Savarese et al. This is an open-access article distributed under the terms of the Creative Commons Attribution License, which permits unrestricted use, distribution, and reproduction in any medium, provided the original author and source are credited.

Funding: No current external funding sources for this study.

Competing Interests: The authors have declared that no competing interests exist.

*E-mail: fpperron@unina.it

\section{Introduction}

Chronic Heart Failure (HF) represents a raising health care concern in developed and developing countries, reaching epidemic proportions [1]. About 1 to $2 \%$ of adult population in developed countries suffers $\mathrm{HF}$, with $\geq 10 \%$ prevalence among elderly $(>70$ years) [2]. At least half of HF patients have reduced left ventricular ejection fraction, and coronary artery disease is the leading cause of chronic HF. Although in recent years progresses of pharmacologic and non-pharmacologic therapies led to substantial improvement of survival and rate of hospitalization in HF patients, prognosis remains poor [2-4].

Recommended pharmacological treatments in chronic HF include angiotensin-converting enzyme inhibitors or angiotensin receptor blockers, beta-adrenergic blockers, loop diuretics and aldosterone antagonists, that improve outcomes at doses used in randomized clinical trials [2]. In clinical practice, dose titration of these drugs is usually driven by assessment of patients' clinical and volume status. However, up-titration of medications in chronic $\mathrm{HF}$ remains suboptimal in clinical practice, with administered doses often lower than those utilized in clinical trials, preventing achievement of the full benefit of evidence-based therapies $[5,6]$. Thus, development of strategies to enhance adherence to guidelines recommended doses of drugs would be much needed to reduce the burden of mortality and morbidity in chronic HF patients.

Measurement of plasma concentrations of B-type natriuretic peptide (BNP) or N-terminal pro-B-type natriuretic peptide (NTproBNP) is useful to rule-out diagnosis and to predict prognosis of HF patients [7]. In addition, several studies demonstrated that 


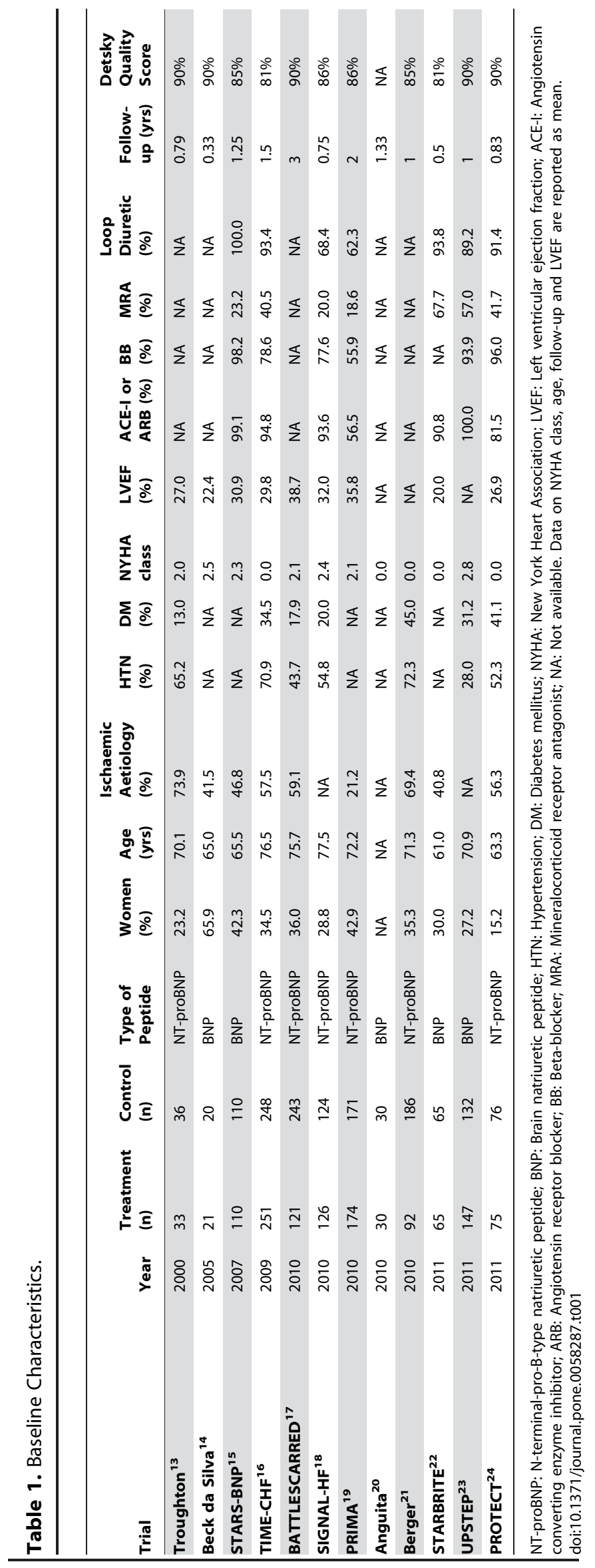


reduction in natriuretic peptide levels reflects the effect of therapy on cardiac loading conditions [8-12]. From these premises, randomized clinical trials [13-24] have evaluated whether adjustment of therapy to achieve pre-specified levels of natriuretic peptide levels, compared to conventional strategy mostly based on assessment of clinical status, results in more favourable mortality/ morbidity in chronic HF patients. However, these trials, and two previous meta-analyses [25,26], including some of them [13$20,22]$, collected a small number of patients, leaving uncertain the role of this strategy in HF patient management $[2,27]$.

Therefore, the aim of this study was to investigate, in an updated meta-analysis including more recent clinical trials, whether a strategy of cardiac peptide-guided therapy, compared to clinically-guided therapy, favourably affects mortality and morbidity in patients with chronic HF.

\section{Materials and Methods}

\section{Data Sources and Searches}

This study was designed according to the PRISMA (Preferred Reporting Items for Systematic reviews and Meta-Analyses) statement, as previously reported from our group [28-31]. MEDLINE, Cochrane, ISI Web of Sciences and SCOPUS databases were searched for articles published in all languages until August 2012.

\section{Study Selection}

Trials were identified by the following headings: NT-proBNPguided, BNP-guided and randomized. As example for MEDLINE the following search was performed: "Natriuretic Peptide, Brain" OR "NT-proBNP") AND "guided" AND "Controlled Clinical Trials, Randomized". Additionally, we searched reference lists of retrieved articles, bibliographies of selected trials, recent reviews and guidelines as well information from colleagues to identify additional eligible studies. Inclusion criteria for a study to be included were as follows: comparison of BNP or NT-proBNP-

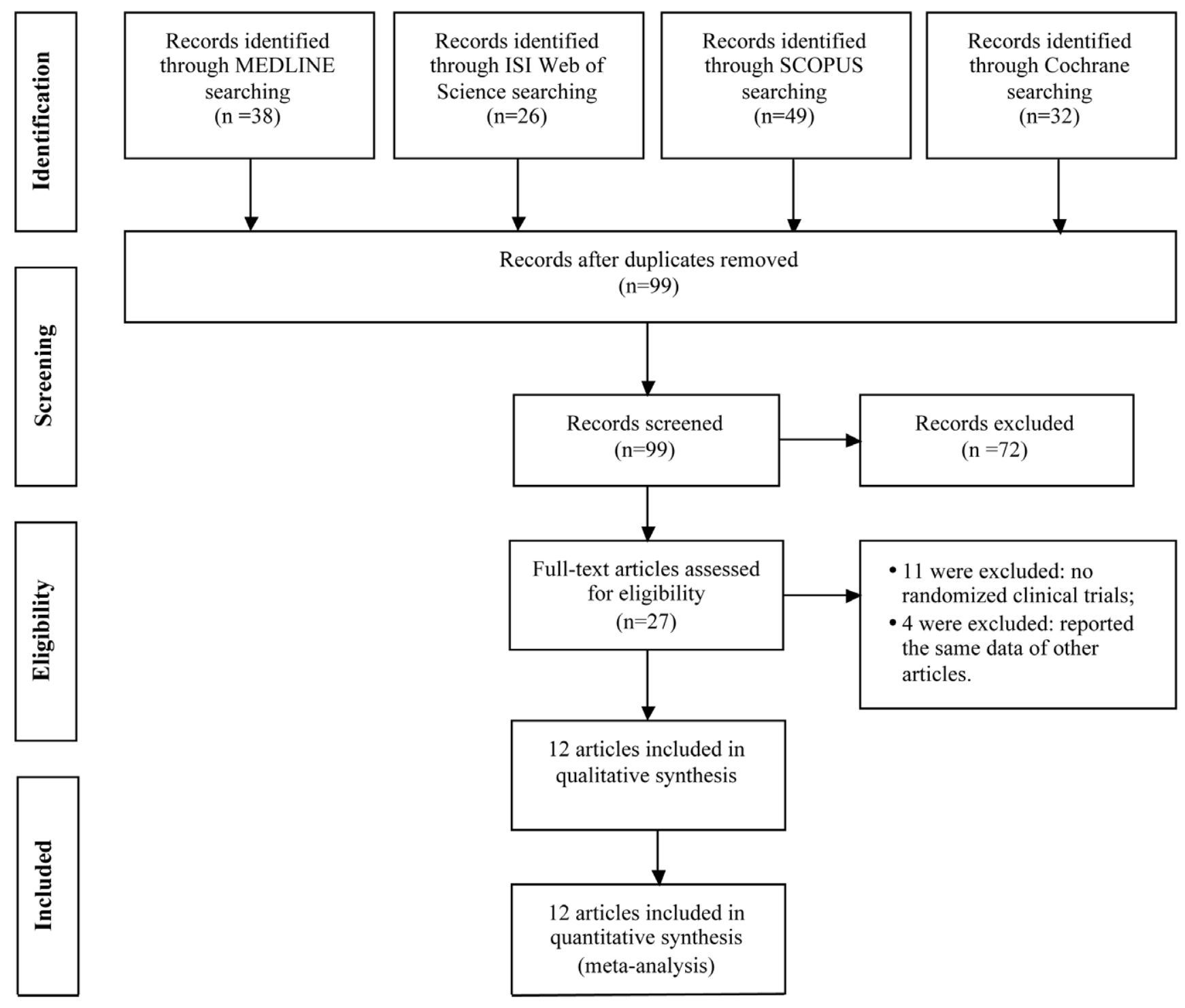

Figure 1. Flow chart showing the progress through the stages of the meta-analysis. doi:10.1371/journal.pone.0058287.g001 
Table 2. Baseline characteristics in NT-proBNP- and BNPguided therapy treatment groups.

\begin{tabular}{lll}
\hline & & \\
\hline & BNP & NT-proBNP \\
\hline Treatment, No. & 373 & 872 \\
\hline Control, No. & 357 & 1084 \\
\hline Follow-up, mean (SD), years & $0.9 \pm 0.4$ & $1.4 \pm 0.8$ \\
Women, No. (\%) & $235(35.1)$ & $660(33.7)$ \\
Age, mean (SD), years & $65.6(4.1)$ & $72.3(4.9)$ \\
Ischaemic Aetiology, No. (\%) & $173(44.2)$ & $904(46.2)$ \\
Hypertension, No. (\%) & NA & $975(49.8)$ \\
Diabetes Mellitus, No. (\%) & NA & $483(24.7)$ \\
NYHA Class, mean (SD) & $2.5(0.4)$ & $2.2(0.2)$ \\
\hline LVEF, mean (SD),(\%) & $24(5.7)$ & $31.7(4.7)$ \\
ACE-Is or ARBs, No. (\%) & $615(96.6)$ & $1025(91.5)$ \\
BBs, No. (\%) & $478(96.0)$ & $924(77.0)$ \\
ARAs, No. (\%) & $298(49.3)$ & $379(30.2)$ \\
\hline Loop Diuretics, No. (\%) & $591(94.4)$ & $990(78.9)$ \\
Detsky Quality Score & $86 \%$ & $87 \%$ \\
\hline & & \\
\hline
\end{tabular}

NT-proBNP: N-terminal-pro-B-type natriuretic peptide; BNP: Brain natriuretic peptide; NYHA: New York Heart Association; LVEF: Left ventricular ejection; ACEI: Angiotensin converting enzyme inhibitor; ARB: Angiotensin receptor blocker; BB: Beta-blocker; ARA: Aldosterone receptor antagonist; NA: Not available.

doi:10.1371/journal.pone.0058287.t002

guided therapy versus a control group in chronic HF patients; randomized protocol; report of end-points (all-cause mortality and all-cause or HF hospitalization).

\section{Data Extraction and Quality Assessment}

Two reviewers independently screened and selected potentially eligible trials according to the inclusion criteria. Two reviewers independently read the full-text of retained studies, which were checked to avoid inclusion of data published in duplicate. Discrepancies were resolved by discussion and consensus. Data on baseline characteristics, presence of diabetes mellitus, hypertension, aetiology of HF, NYHA (New York Heart Association) class, HF therapy and pre-specified outcomes, including all-cause mortality and all-cause or HF hospitalization. Trials' quality was evaluated by Detsky method; studies scoring $<50 \%$ were considered to be of low quality, those with a score of $>75 \%$ were deemed to be of high quality, those with a score of $\geq 50 \%$ and $<75 \%$ were designated to be of moderate quality [32](Table 1).

Of 145 articles identified by the initial search, 72 were excluded by title and 27 were retrieved for more detailed evaluation. Afterwards 15 studies were excluded (for instance no randomized clinical trials or reporting the same data of other articles) and the presence of the same data published in duplicate papers was resolved choosing the article reporting more information. Therefore, 12 were included in meta-analysis (Figure 1). Included trials and populations' details are listed in Table 1. Five studies compared BNP-guided therapy to usual care [14,15,20,22,23] and 7 compared NT-proBNP-guided therapy to usual care $[13,16-19,21,24]$.

\section{Data Synthesis and Analysis}

Odds ratios (OR) of the effect of randomized treatments were calculated using the metan routine (STATA Statacorp, version 11.0) [33]. OR and 95\% Confidence Interval (CI) for each outcome were separately calculated for each trial, with grouped data, using the intention-to-treat principle [34]. The choice to use ORs was driven by the retrospective design of the meta-analysis, based on published studies that vary in design, subjects' population, treatment regimen, primary outcome measure and quality [35]. Pooled ORs were logarithmically transformed and weighted for the inverse of variance. Overall estimates of effect were calculated with a fixed-effects, random effects model or Peto's method [36] when appropriate. The assumption of homogeneity between the treatment effects in different trials was tested by $\mathcal{Q}$ statistic and further quantified by $\mathrm{I}^{2}$ statistic. A significant heterogeneity was defined by a $\mathrm{p} \leq 0.10$ at $\mathrm{Q}$ statistic and by $\mathrm{I}^{2}>30 \%$, whereas $\mathrm{I}^{2}<40 \%$ might indicate a not important heterogeneity. The significance level for the overall estimates of effect and for meta-regression analyses was set at $\mathrm{p} \leq 0.05$. The first objective of the study was to separately investigate the effects of peptide-guided therapy on all-cause mortality and on HF-related hospitalization. Additionally, we investigated the effects of peptideguided therapy on all-cause hospitalization and the effect of age on peptide-guided therapy using a composite outcome including allcause mortality and HF-related hospitalization. Finally, we sought to assess differences between BNP- vs NT-proBNP-guided therapy on each of the above outcomes.

\section{Sensitivity Analysis}

To explore the influence of potential effect modifiers on results, meta-regression analyses were performed with the metareg command [37](STATA Statacorp, version 11.0) to test demographic characteristics of the study population, percent of patients with DM, percent of patients with hypertension, percent of patients with ischaemic-related HF, current therapy, length of follow-up, year of publication and quality of trials [32]. For all meta-regression analyses, random effects model was used [38]. To estimate the additive (between-study) component of variance tau- 2 the restricted maximum likelihood (REML) method was used to take into account the occurrence of residual heterogeneity, not explained by the potential effect modifiers [38]. To verify the consistency of the results, the influence of individual studies on the summary effect estimate (one study removed meta-analysis) was assessed using the metaninf command (STATA Statacorp, version 11.0) [39].

\section{Publication Bias}

To evaluate potential publication bias, a weighted linear regression was used, with the natural $\log$ of the OR as the dependent variable and the inverse of the total sample size as the independent variable. This is a modified Macaskill's test, that gives more balanced type I error rates in the tail probability areas in comparison to other publication bias tests [40].

\section{Results}

\section{Characteristics of Included Trials}

Baseline characteristics of 12 trials included in the meta-analysis are shown in Table 1. Of 2,686 patients, 730 were enrolled in trials comparing BNP-guided therapy to usual care and 1,956 in trials comparing NT-proBNP-guided therapy to usual care. Mean follow-up duration was $1.2 \pm 0.7$ years. The overall mean age of subjects was $70 \pm 6$ years and $33 \%$ were women. Characteristics of patients enrolled in BNP or in NT-proBNP trials are reported in Table 2. 


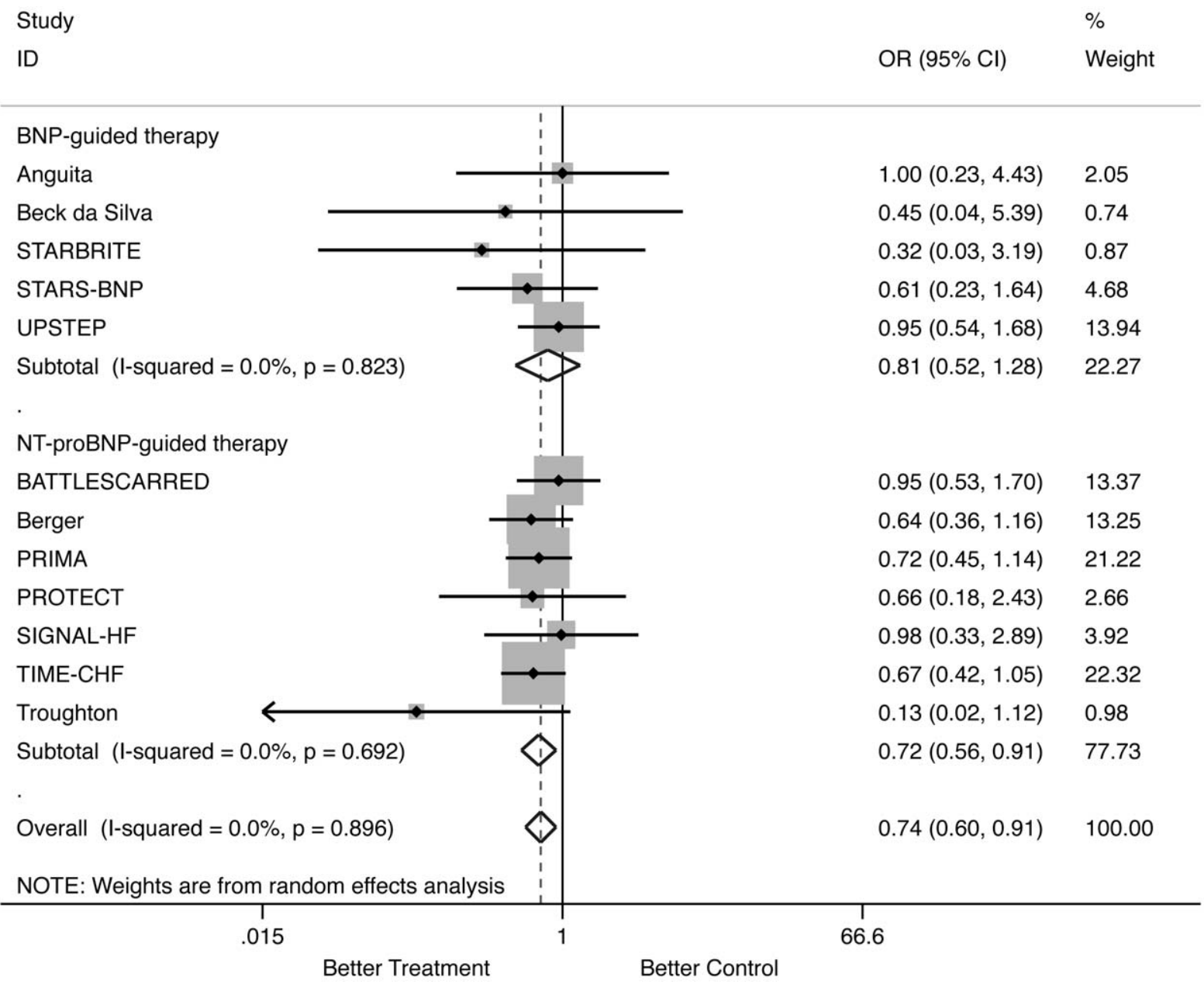

Figure 2. Odds ratios of all-cause mortality. Solid squares represent odds ratios in trials and have a size proportional to the number of events. The $95 \%$ confidence intervals for individual trials are denoted by lines and those for the pooled odd ratios by empty diamonds. doi:10.1371/journal.pone.0058287.g002

\section{Outcomes Analysis}

All-cause mortality (Figure 2). Natriuretic peptide-guided therapy (using either BNP- or NT-proBNP-guided) led to a significant reduction of all-cause mortality (OR: 0.738; 95\% CI: 0.596 to 0.913 ; comparison $\mathrm{p}=0.005$; heterogeneity $\mathrm{p}=0.896$ ) without heterogeneity among studies. When separately analyzed, NT-proBNP-guided therapy significantly reduced all-cause mortality (OR: 0.717 ; 95\% CI: 0.563 to 0.914 ; comparison $\mathrm{p}=0.007$; heterogeneity $\mathrm{p}=0.692)$, whereas $\mathrm{BNP}$-guided therapy did not (OR: $0.814 ; 95 \%$ CI: 0.518 to 1.279 ; comparison $\mathrm{p}=0.371$; heterogeneity $\mathrm{p}=0.823$ ).

HF-related hospitalization (Figure 3). Natriuretic peptideguided therapy led to a significant reduction of HF-related hospitalization (OR: 0.554; 95\% CI: 0.399 to 0.769; comparison $p=0.000$; heterogeneity $p=0.019)$. When separately assessed, NT-proBNP-guided therapy significantly reduced HF-related hospitalization (OR: 0.531; 95\% CI: 0.347 to 0.811 ; comparison $\mathrm{p}=0.003$; heterogeneity $\mathrm{p}=0.032)$, whereas BNP-guided therapy did not (OR: 0.599; 95\% CI: 0.303 to 1.187; comparison $p=0.142$; heterogeneity $p=0.045)$. According to the Cochrane Handbook [41], heterogeneity among studies resulting in this analysis was resolved when 2 outlying trials $[15,17]$ were excluded, fully confirming the results (all trials analysis - OR: $0.546,95 \%$ CI: 0.393 to 0.759 , comparison $p=0.000$, heterogeneity $\mathrm{p}=0.151 ; \mathrm{NT}$-proBNP analysis - OR: $0.459,95 \%$ CI: 0.319 to 0.661 , comparison $\mathrm{p}=0.000$, heterogeneity $\mathrm{p}=0.240$; BNP analysis - OR: $0.819,95 \%$ CI: 0.528 to 1.269 , comparison $\mathrm{p}=0.371$, heterogeneity $\mathrm{p}=0.689$ ).

All-cause hospitalization (Figure 4). Natriuretic peptideguided therapy did not reduce significantly all-cause hospitalization (OR: 0.803; 95\% CI: 0.629 to 1.024; comparison $\mathrm{p}=0.077$; heterogeneity $\mathrm{p}=0.604$ )(Figure 3 ) without heterogeneity among studies. When separately assessed, neither NT-proBNP-guided therapy (OR: 0.779 ; 95\% CI: 0.414 to 1.465; comparison $p=0.438$; heterogeneity $p=0.181$, nor BNP-guided therapy (OR: 0.726 ; 95\% CI: 0.509 to 1.035 ; comparison $\mathrm{p}=0.077$; heterogeneity $\mathrm{p}=0.836$ ) significantly reduced all-cause hospitalization. For this outcome, the effect was dominated by TIMECHF trial [16], since after its removal the reduction of all-cause hospitalization determined by natriuretic peptide-guided therapy became significant (OR: $0.689 ; 95 \%$ CI: 0.494 to 0.962 ; $\mathrm{p}=0.029)$.

Younger vs older patients. Separate outcome analyses on patients younger or older than 75 years were performed using data reported in 3 trials $[16,17,23]$. The composite outcome of all-cause mortality and HF-related hospitalization was significantly reduced by natriuretic peptide-guided therapy in younger patients $(\leq 75$ years)(OR: 0.449 ; $95 \%$ CI: 0.207 to $0.973 ; \mathrm{p}=0.043)$, but not in 
Study

ID
$\%$

OR $(95 \% \mathrm{Cl}) \quad$ Weight

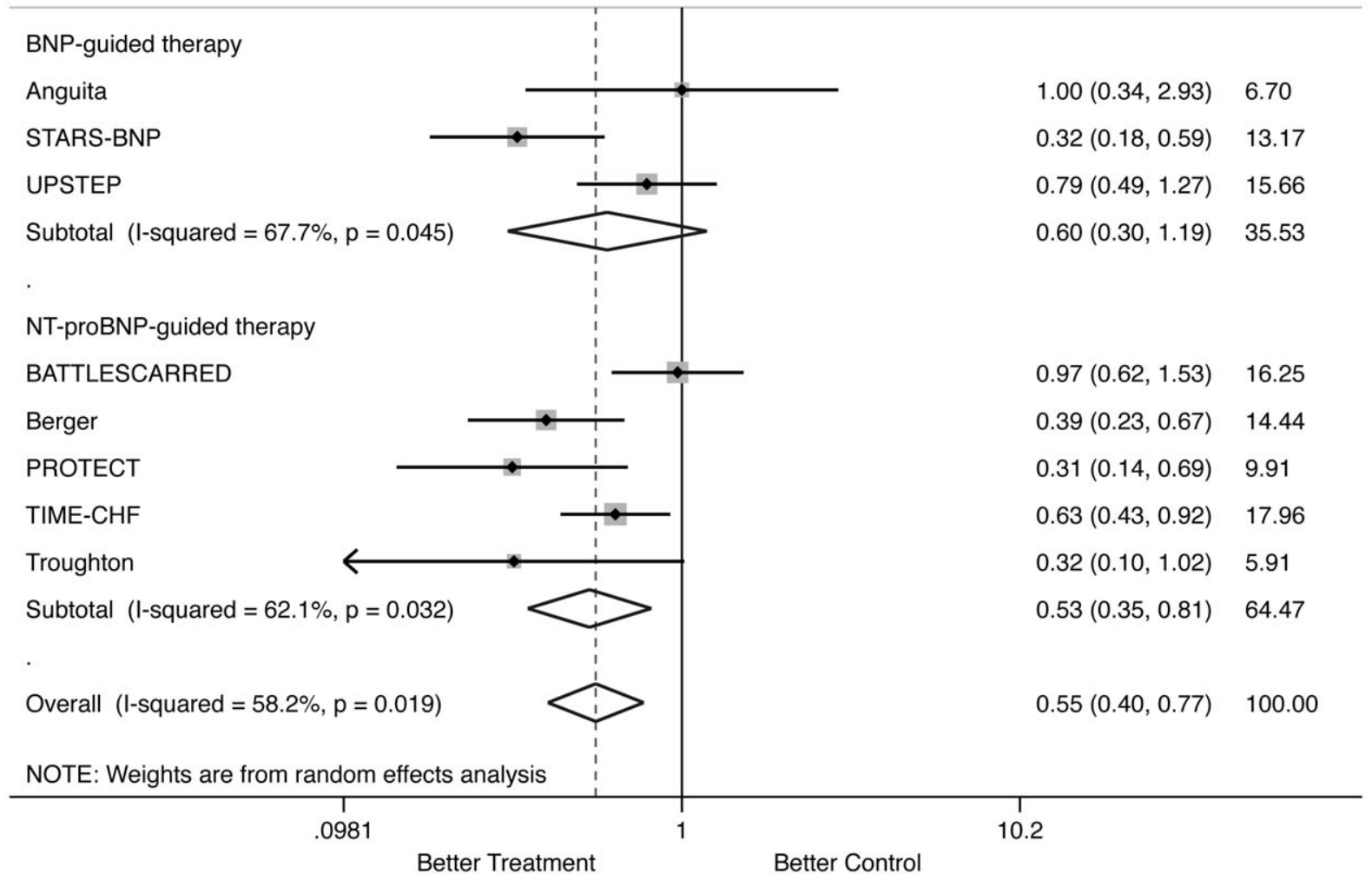

Figure 3. Odds ratios of heart failure-related hospitalization. Solid squares represent odds ratios in trials and have a size proportional to the number of events. The $95 \%$ confidence intervals for individual trials are denoted by lines and those for the pooled odd ratios by empty diamonds. doi:10.1371/journal.pone.0058287.g003

older patients $(>75$ years)(OR: $0.800 ; 95 \%$ CI: 0.423 to 1.513 ; $\mathrm{p}=0.493)$.

\section{Sensitivity Analysis}

Results for each outcome were confirmed when potential effect modifiers were introduced as covariates in the meta-regression analysis (Table 3). Meta-analyses assessing the effect of natriuretic peptide-guided therapy on all-cause mortality and HF-related hospitalization were performed removing each study at a time, and in no cases removal of a single study affected the significance of the results (Figures 5,6). Additionally, removal of TIME-CHF trial [16] made significant the reduction of all-cause hospitalization determined by natriuretic peptide-guided therapy (Figure 7).

\section{Publication Bias}

Macaskill's modified test did not show publication bias for any outcome.

\section{Discussion}

The findings of the present study indicate that, in patients with chronic HF due to systolic dysfunction, adjustment of pharmacologic therapy guided by natriuretic peptide measurements significantly reduces all-cause mortality as well HF-related hospitalization.

\section{Previous Studies}

Natriuretic peptide levels reflect cardiac loading conditions $[42,43]$ and predict adverse cardiac events in patients with asymptomatic or symptomatic $\mathrm{HF}$ [10]. As cardiac natriuretic peptides are simply to obtain an objective marker of disease severity in $\mathrm{HF}$, their role as therapeutic guidance has been investigated in several clinical trials. These studies mainly investigated two potential strategies of using cardiac peptides in the management of HF patients. The first, reported in the recent NorthStar trial [4], assessed whether high-risk but stable chronic $\mathrm{HF}$ patients, identified as those with NT-proBNP levels $>1000 \mathrm{pg} / \mathrm{mL}$, would benefit from prolonged specialized HF clinic assistance compared to referral back to general practitioners, and demonstrated no differences in the composite of mortality and hospitalization for cardiac causes, suggesting that the basal value of cardiac peptides has limited value to select out of hospital management strategy in $\mathrm{HF}$ patients. The second approach consists of targeting pharmacologic therapy on pre-specified levels of cardiac peptides, to optimize the effects of drugs. This approach has received much interest and has been tested in several trials that yielded conflicting results, with some studies demonstrating mortality or morbidity benefit from peptide-guided therapy $[13,15,21,24]$, others reporting benefit only in younger patients $[16,17]$ or only in responder patients [23], and other studies showing no advantages of peptide-guided compared to clinicallyguided therapy [14,18-20,22]. To overcome uncertainty produced by conflicting results of single studies, 2 previous meta-analyses 
Study

ID
$\%$

OR $(95 \% \mathrm{Cl}) \quad$ Weight

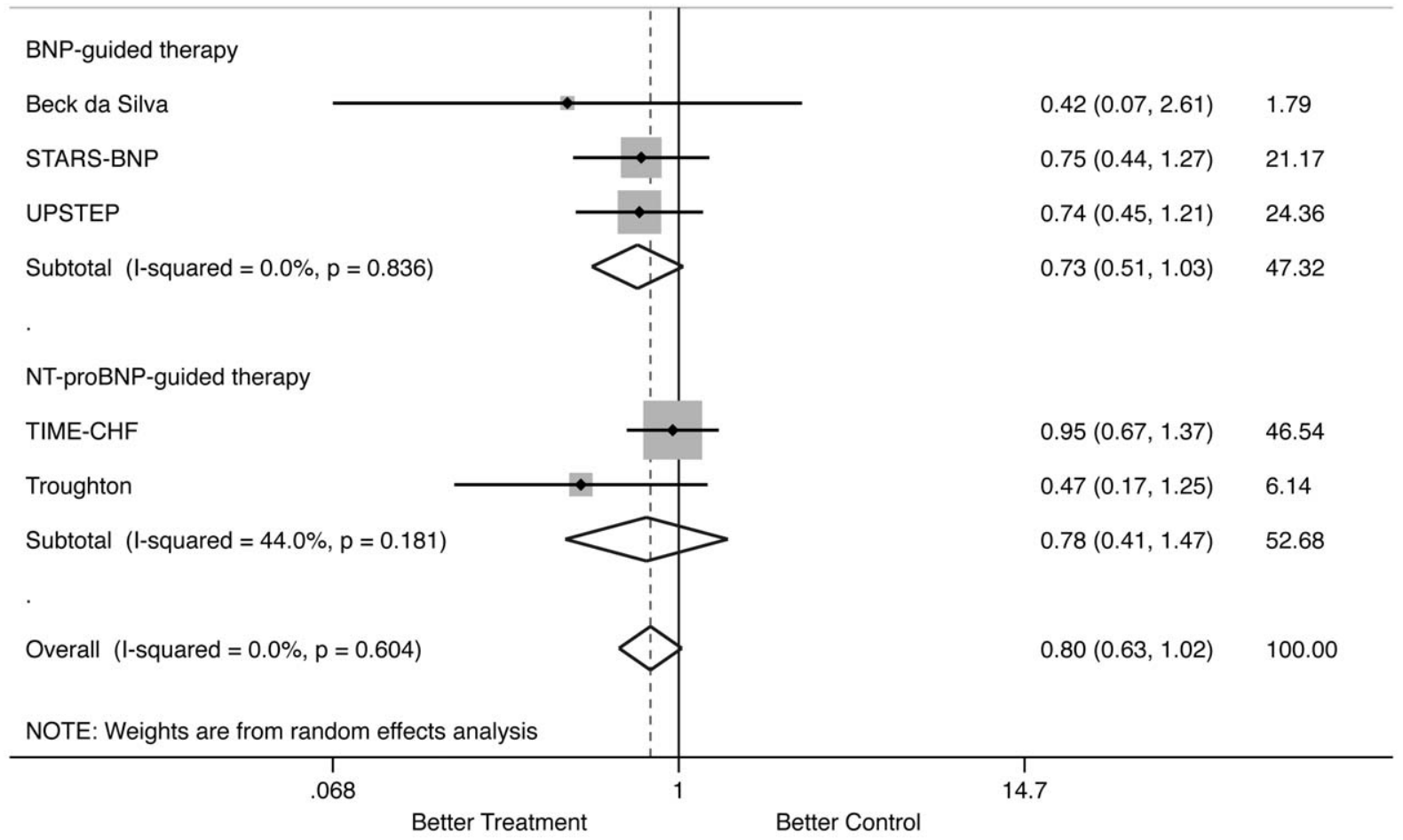

Figure 4. Odds ratios of all-cause hospitalization. Solid squares represent odds ratios in trials and have a size proportional to the number of events. The $95 \%$ confidence intervals for individual trials are denoted by lines and those for the pooled odd ratios by empty diamonds. doi:10.1371/journal.pone.0058287.g004

investigated the usefulness of natriuretic peptide-guided therapy in chronic HF $[25,26]$. These meta-analyses, collecting 6 [13,15$17,19,22]$ or 8 [13-20] randomized clinical trials, reported non definitive results on all-cause mortality and no benefit on hospitalization afforded by peptide-guided therapy, leading the recent European Society of Cardiology Guidelines on chronic HF [2] to consider still uncertain and without recommendations the use of cardiac peptides to assist management of patients, and the AHA/ACG HF Guidelines [27] to give a low-level of recommen-

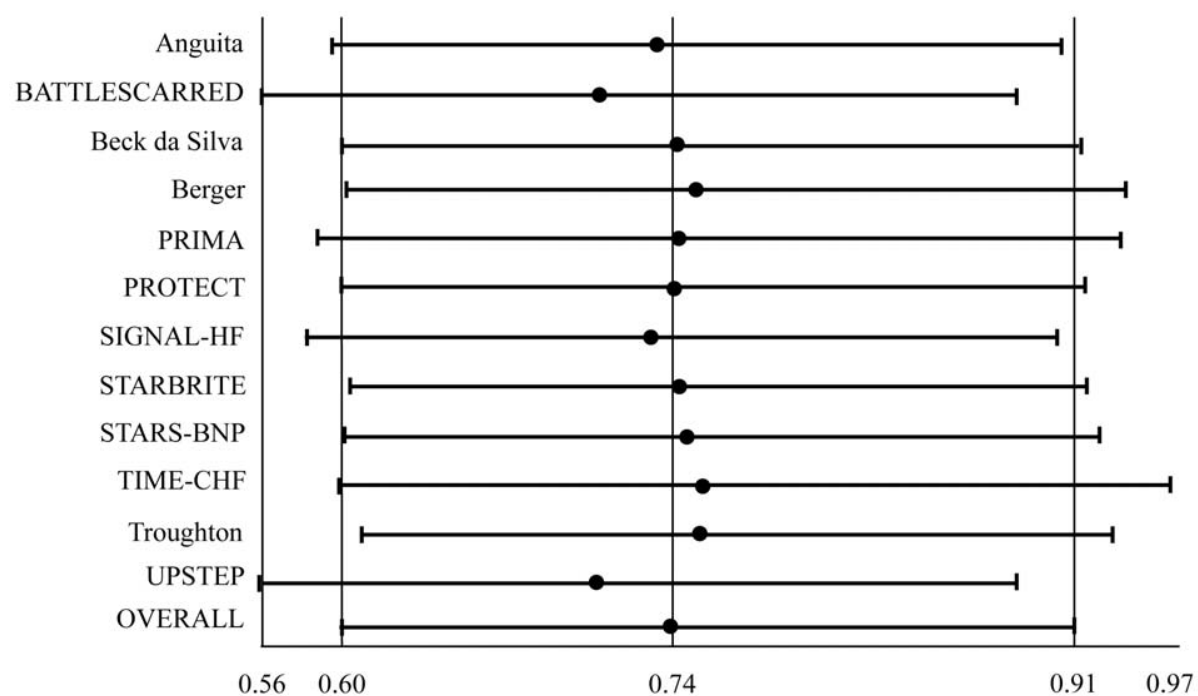

Figure 5. One study removed analysis for all-cause mortality. Rows represent the results of meta-analysis of all studies except the omitted study named in that row.

doi:10.1371/journal.pone.0058287.g005 


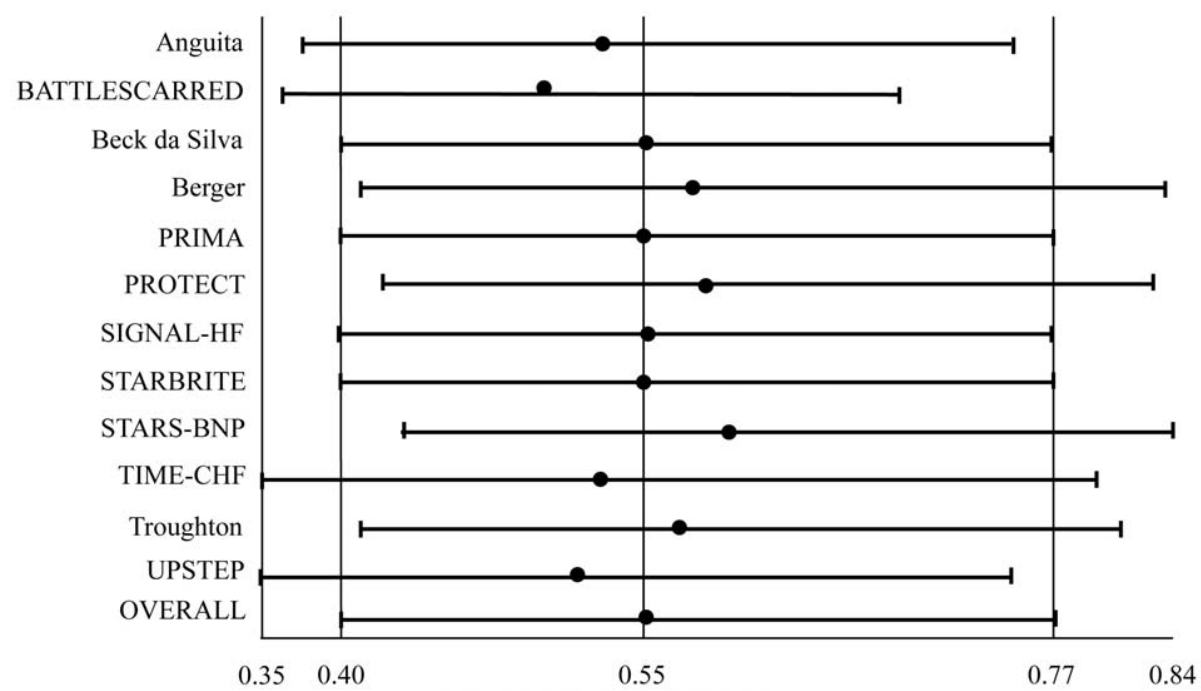

Figure 6. One study removed analysis for heart failure-related hospitalization. Rows represent the results of meta-analysis of all studies except the omitted study named in that row. doi:10.1371/journal.pone.0058287.g006

dation (IIb) to peptide-guided HF therapy, with both guidelines soliciting additional evidence.

In fact, previous meta-analyses substantially differ from the current one. In the meta-analysis by Felker et al. [25] only 6 studies were collected reporting 1,627 patients. Although a significant benefit on all-cause mortality in patients assigned to peptideguided therapy was reported, the analysis was limited by the inclusion of 3 still unpublished studies, which prevented a detailed collection of patients' population characteristics. Besides, the effects on all-cause or HF-related hospitalization were not analyzed. The more recent and largest meta-analysis by Porapakkham et al. [26] included 8 studies in 1,726 patients. In this analysis the favorable effect on all-cause mortality in patients assigned to peptide-guided therapy was mostly driven by the TIME-CHF trial [16], as in the sensitivity analysis the statistical significance of the effect was lost when this trial, but not any other trial included in that meta-analysis, was removed from the analysis. Notably, no difference was observed in all-cause or HFrelated hospitalization. Moreover, in both previous meta-analyses $[25,26]$, no separate analysis for BNP- or NT-proBNP-guided therapy was performed.

Thus the current meta-analysis substantially adds to previous studies for several aspects. First, compared to previous metaanalysis, it provides evidence of benefit from larger number of studies (12 instead of 8 ) and of patients (2,686 vs 1,726), including more recent clinical trials in which up to date optimized pharmacologic HF therapy was used. In addition, this metaanalysis for the first time reports a clear and substantial benefit in HF-related hospitalization, which represent the main morbidity outcome in HF patients, profoundly interfering with quality of life as well with health cost expenditure. Third, the mortality benefit observed was quite consistent and not influenced, in sensitivity

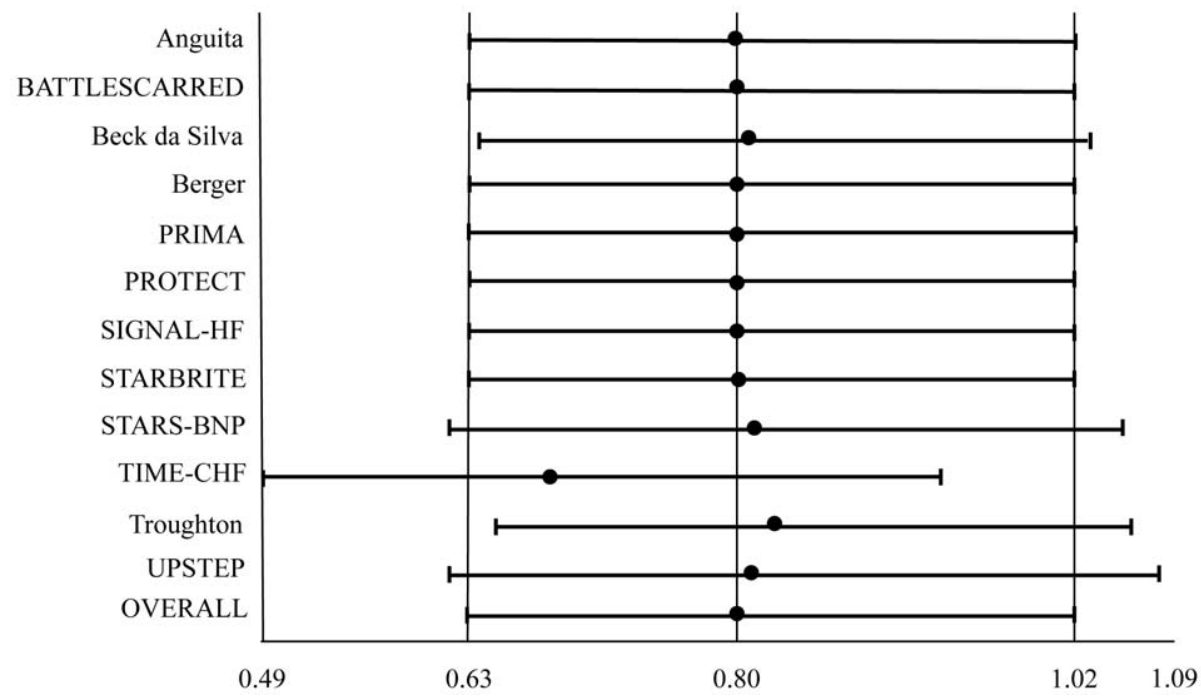

Figure 7. One study removed analysis for all-cause hospitalization. Rows represent the results of meta-analysis of all studies except the omitted study named in that row. doi:10.1371/journal.pone.0058287.g007 
Table 3. Influence analysis of potential effect modifiers on the outcomes.

\begin{tabular}{|c|c|c|c|c|c|c|}
\hline & \multicolumn{2}{|c|}{ All-cause mortality } & \multicolumn{2}{|c|}{ HF-related hospitalization } & \multicolumn{2}{|c|}{ All-cause hospitalization } \\
\hline & Tau & $P$ value & Tau & $P$ value & Tau & $P$ value \\
\hline Year of publication & 1.76 & 0.108 & 1.21 & 0.271 & 1.03 & 0.379 \\
\hline Women & -0.30 & 0.768 & 0.41 & 0.696 & 0.05 & 0.964 \\
\hline Age & 0.72 & 0.488 & 2.38 & 0.064 & 1.14 & 0.338 \\
\hline Ischaemic Aetiology & -0.23 & 0.828 & -0.02 & 0.987 & -0.30 & 0.789 \\
\hline NYHA Class & 0.68 & 0.525 & 0.43 & 0.709 & NA & NA \\
\hline LVEF & 1.35 & 0.219 & 2.06 & 0.132 & 1.05 & 0.406 \\
\hline Follow-up & 0.76 & 0.464 & 1.97 & 0.096 & 1.53 & 0.223 \\
\hline ACE-Is or ARBs & 0.24 & 0.821 & 0.84 & 0.491 & NA & NA \\
\hline BBs & 0.31 & 0.769 & -0.85 & 0.487 & NA & NA \\
\hline ARAs & 0.30 & 0.773 & 1.84 & 0.207 & NA & NA \\
\hline Loop Diuretics & -0.25 & 0.814 & -1.33 & 0.314 & NA & NA \\
\hline DM & -0.73 & 0.498 & -1.82 & 0.143 & NA & NA \\
\hline Hypertension & -1.37 & 0.230 & -1.33 & 0.255 & NA & NA \\
\hline Detsky Quality Score & 1.14 & 0.283 & 1.13 & 0.310 & -0.90 & 0.435 \\
\hline
\end{tabular}

NYHA: New York Heart Association; LVEF: Left ventricular ejection; ACE-I: Angiotensin converting enzyme inhibitor; ARB: Angiotensin receptor blocker; BB: Beta-blocker; MRA: Mineralocorticoid receptor antagonist; DM: Diabetes mellitus; NA: not available.

doi:10.1371/journal.pone.0058287.t003

analysis, by any single study or by any potential confounders, which, together with the lack of significant heterogeneity, significantly strengthens the robustness of the result. Similarly to previous meta-analysis [26], we also observed no significant benefit in elderly patients, analyzing elderly subgroups from 3 trials $[16,17,23]$. Although it is conceivable that more frequent presence of comorbidities [44] may prevent or even make potentially harmful up-titration of HF drugs in elderly patients, this finding should be interpreted with caution as it comes from subgroup analysis of only 3 trials. However, use of actual rather tan agestratified values may also have influenced the results in the elderly group [45]. Finally, at difference with previous meta-analyses, the higher number of patients included in our study allowed to separately investigate the effects of BNP- and of NT-proBNPguided therapy, suggesting that NT-proBNP- but not BNP-guided therapy was significantly associated to improved survival as well reduced hospitalization. This finding could be explained by more favorable characteristics of NT-proBNP compared to BNP, including higher circulatory levels and longer stability, as well as reduced in vitro degradation [46].

\section{Limitations}

There are limitations to this study that need to be acknowledged. First, it was not a patient-level but an aggregate data metaanalysis. Second, the findings of the separate analysis for BNP- and NT-proBNP-guided therapy need to be interpreted with caution since no single trial was designed to head-to-head compare BNPvs NT-proBNP-guided therapy. Besides, patients enrolled in BNP trials had lower average ejection fraction compared to NTproBNP and were better treated, possibly indicating a sicker population, with reduced room for drug up-titration. Finally patients enrolled in NT-proBNP trials were more than twofold compared to those enrolled in BNP trials, which may have

\section{References}

1. Fang J, Mensah GA, Croft JB, Keenan NL (2008) Heart failure-related hospitalization in the U.S., 1979 to 2004. J Am Coll Cardiol 52: 428-434. prevented to observe significant association in the latters. Thus, our findings are in this regard provisional and deserve further investigation in ad hoc designed trials. In addition, although the findings of the study indicate that natriuretic peptide-guided therapy is associated with outcome benefits, the target peptide level to reach cannot be defined from the current findings. Finally, the possibility that our findings were influenced by more aggressive pharmacologic therapy in randomized to study compared to control arms cannot be excluded, although it does not detract the relevance of the results. However, there are also strengths of the current study. In fact, at variance with previous studies $[25,26]$, use of a random rather than fixed-effect model and of ORs instead of Relative Risk, made more rigorous the statistical analysis and strengthened its robustness.

\section{Conclusions}

Use of cardiac peptides (BNP or NT-proBNP) to guide pharmacologic therapy in patients with chronic $\mathrm{HF}$ is associated with a significant reduction of mortality and HF-related hospitalization, especially in patients younger than 75 years. In particular, NT-proBNP-guided therapy is associated with reduced all-cause mortality and HF-related hospitalization but not all-cause hospitalization, whereas BNP-guided therapy is not significantly associated with reduced mortality and morbidity. The findings of the present study may be of help for defining the role of this approach in $\mathrm{HF}$ guidelines and in clinical practice.

\section{Author Contributions}

Collected Data: GS MP SD BT FG DL GR PPF. Conceived and designed the experiments: GS PPF. Analyzed the data: GS PPF. Wrote the paper: GS PPF. Auricchio A, et al. (2012) ESC Guidelines for the diagnosis and treatment of 
acute and chronic heart failure 2012: The Task Force for the Diagnosis and Treatment of Acute and Chronic Heart Failure 2012 of the European Society of Cardiology. Developed in collaboration with the Heart Failure Association (HFA) of the ESC. Eur J Heart Fail 14: 803-869.

3. Stewart S, MacIntyre K, Hole DJ, Capewell S, McMurray JJ (2001) More 'malignant' than cancer? Five-year survival following a first admission for heart failure. Eur J Heart Fail 3: 315-322.

4. Schou M, Gustafsson F, Videbaek L, Tuxen C, Keller N, et al. (2012) Extended heart failure clinic follow-up in low-risk patients: a randomized clinical trial (NorthStar). Eur Heart J. doi:10.1093/eurheartj/ehs235.

5. Komajda M, Lapuerta P, Hermans N, Gonzalez-Juanatey JR, van Veldhuisen DJ, et al. (2005) Adherence to guidelines is a predictor of outcome in chronic heart failure: the MAHLER survey. Eur Heart J 26: 1653-1659.

6. Powell LH, Calvin JE Jr, Richardson D, Janssen I, Mendes de Leon CF, et al. (2010) Self-management counseling in patients with heart failure: the heart failure adherence and retention randomized behavioral trial. JAMA 304: 13311338.

7. Groenning BA, Raymond I, Hildebrandt PR, Nilsson JC, Baumann M, et al. (2004) Diagnostic and prognostic evaluation of left ventricular systolic heart failure by plasma Nterminal pro-brain natriuretic peptide concentrations in a large sample of the general population. Heart 90: 297-303.

8. White M, Rouleau JL, Afzal R, Floras J, Yusuf S, et al. (2009) Effects of enalapril, candesartan or both on neurohumoral activation and $\mathrm{LV}$ volumes and function in patients with heart failure not treated with a beta-blocker. Ther Adv Cardiovasc Dis 3: 113-121.

9. Iraqi W, Rossignol P, Angioi M, Fay R, Nuée J, et al. (2009) Extracellular cardiac matrix biomarkers in patients with acute myocardial infarction complicated by left ventricular dysfunction and heart failure: insights from the Eplerenone Post-Acute Myocardial Infarction Heart Failure Efficacy and Survival Study (EPHESUS) study. Circulation 119: 2471-2479.

10. Hartmann F, Packer M, Coats AJ, Fowler MB, Krum H, et al. (2004) Prognostic impact of plasma N-terminal pro-brain natriuretic peptide in severe chronic congestive heart failure: a substudy of the Carvedilol Prospective Randomized Cumulative Survival (COPERNICUS) trial. Circulation 110: 1780-1786.

11. Berry C, Murphy NF, De Vito G, Galloway S, Seed A, et al. (2007) Effects of aldosterone receptor blockade in patients with mild-moderate heart failure taking a betablocker. Eur J Heart Fail 9: 429-434.

12. Adams KF Jr, Felker GM, Fraij G, Patterson JH, O'Connor CM (2010) Biomarker guided therapy for heart failure: focus on natriuretic peptides. Heart Fail Rev 15: 351-370.

13. Troughton RW, Frampton CM, Yandle TG, Espiner EA, Nicholls MG, et al. (2000) Treatment of heart failure guided by plasma aminoterminal brain natriuretic peptide (N-BNP) concentrations. Lancet 355: 1126-1130.

14. Beck-da-Silva L, de Bold A, Fraser M, Williams K, Haddad H (2005) BNPguided therapy not better than expert's clinical assessment for beta-blocker titration in patients with heart failure. Congest Heart Fail 11: 248-253.

15. Jourdain P, Jondeau G, Funck F, Gueffet P, Le Helloco A, et al. (2007) Plasma brain natriuretic peptide-guided therapy to improve outcome in heart failure: the STARS-BNP Multicenter Study. J Am Coll Cardiol 49: 1733-1739.

16. Pfisterer M, Buser P, Rickli H, Gutmann M, Erne P, et al. (2009) BNP-guided vs symptom-guided heart failure therapy: the Trial of Intensified vs Standard Medical Therapy in Elderly Patients With Congestive Heart Failure (TIMECHF) randomized trial. JAMA 301: 383-392.

17. Lainchbury JG, Troughton RW, Strangman KM, Frampton CM, Pilbrow A, et al. (2009) N-terminal pro-B-type natriuretic peptide-guided treatment for chronic heart failure: results from the BATTLESCARRED (NT-proBNPAssisted Treatment To Lessen Serial Cardiac Readmissions and Death) trial. J Am Coll Cardiol 55: 53-60.

18. Persson H, Erntell H, Eriksson B, Johansson G, Swedberg K, et al. (2010) Improved pharmacological therapy of chronic heart failure in primary care: a randomized Study of NT-proBNP Guided Management of Heart FailureSIGNAL-HF (Swedish Intervention study-Guidelines and NT-proBNP AnaLysis in Heart Failure). Eur J Heart Fail 12: 1300-1308.

19. Eurlings LW, van Pol PE, Kok WE, van Wijk S, Lodewijks-van der Bolt G, et al. (2010) Management of chronic heart failure guided by individual N-terminal pro-B-type natriuretic peptide targets: results of the PRIMA (Can PRo-brainnatriuretic peptide guided therapy of chronic heart failure IMprove heart fAilure morbidity and mortality?) study. J Am Coll Cardiol 56: 2090-2100.

20. Anguita M, Esteban F, Castillo JC, Mazuelos F, López-Granados A, et al. (2010) Usefulness of brain natriuretic peptide levels, as compared with usual clinical control, for the treatment monitoring of patients with heart failure. Med Clin. (Barc) 135: 435-440.

21. Berger R, Moertl D, Peter S, Ahmadi R, Huelsmann M, et al. (2010) N-terminal pro-B-type natriuretic peptide-guided, intensive patient management in addition to multidisciplinary care in chronic heart failure a 3-arm, prospective, randomized pilot study. J Am Coll Cardiol 55: 645-653.
22. Shah MR, Califf RM, Nohria A, Bhapkar M, Bowers M, et al. (2011) The STARBRITE trial: a randomized, pilot study of B-type natriuretic peptideguided therapy in patients with advanced heart failure. J Card Fail 17: 613-621.

23. Karlström P, Alehagen U, Boman K, Dahlström U; UPSTEP-study group (2011) Brain natriuretic peptide-guided treatment does not improve morbidity and mortality in extensively treated patients with chronic heart failure: responders to treatment have a significantly better outcome. Eur J Heart Fail 13: $1096-1103$.

24. Januzzi JL Jr, Rehman SU, Mohammed AA, Bhardwaj A, Barajas L, et al. (2011) Use of amino-terminal pro-B-type natriuretic peptide to guide outpatient therapy of patients with chronic left ventricular systolic dysfunction. J Am Coll Cardiol 58: 1881-1889.

25. Felker GM, Hasselblad V, Hernandez AF, O'Connor CM (2009) Biomarkerguided therapy in chronic heart failure: a meta-analysis of randomized controlled trials. Am Heart J 158: 422-430.

26. Porapakkham P, Porapakkham P, Zimmet H, Billah B, Krum H (2010) B-type natriuretic peptide-guided heart failure therapy: A meta-analysis. Arch Intern Med 170: 507-514.

27. Jessup M, Abraham WT, Casey DE, Feldman AM, Francis GS, et al. (2009) 2009 focused update: ACCF/AHA Guidelines for the Diagnosis and Management of Heart Failure in Adults: a report of the American College of Cardiology Foundation/American Heart Association Task Force on Practice Guidelines: developed in collaboration with the International Society for Heart and Lung Transplantation. Circulation 119: 1977-2016.

28. Moher D, Liberati A, Tetzlaff J, Altman DG; PRISMA Group (2009) Preferred reporting items for systematic reviews and meta-analyses: the PRISMA statement. J Clin Epidemiol 62: 1006-1012.

29. Costanzo P, Perrone-Filardi P, Petretta M, Marciano C, Vassallo E, et al. (2009) Calcium channel blockers and cardiovascular outcomes: a meta-analysis of 175,634 patients. J Hypertens 27: 1136-1151.

30. Savarese G, Musella F, Volpe M, Paneni F, Perrone-Filardi P (2012) Effects of atorvastatin and rosuvastatin on renal function: A meta-analysis. Int J Cardiol. doi:10.1016/j.ijcard.2012.05.010.

31. Savarese G, Paolillo S, Costanzo P, D'Amore C, Cecere M, et al. (2012) Do changes of 6-minute walk distance predict clinical events in patients with pulmonary arterial hypertension? J Am Coll Cardiol 60: 1192-1201.

32. Detsky A, Naylor C, O’Rourke K, McGeer A, L’Abbé K (1992) Incorporating variations in the quality of individual randomized trials into meta-analysis. J Clin Epidemiol 45: 255-265.

33. Sharp S 1, Sterne J (1998) Meta-analysis. STB Reprints 7: 100-108.

34. Whitehead A (2002) Meta-analysis of Controlled Clinical Trials. Chichester, England: John Wiley \& Sons Inc.

35. Davies HT, Crombie IK, Tavakoli M (1998) When can odds ratios mislead? BMJ 316: 989-991.

36. Bradburn MJ, Deeks JJ, Berlin JA, Russell Localio A (2007) Much ado about nothing: a comparison of the performance of meta-analytical methods with rare events. Stat Med 26: 53-77.

37. Sharp SJ (1998) Meta-analysis regression. STB 42: 16-22.

38. Thompson SG, Sharp SJ (1999) Explaining heterogeneity in meta-analysis: a comparison of methods. Stat Med 18: 2693-2708.

39. Tobias A (1999) Assessing the influence of a single study in meta-analysis. STB 47: 15-17.

40. Peters JL, Sutton AJ, Jones DR, Abrams KR, Rushton L (2006) Comparison of two methods to detect publication bias in meta-analysis. JAMA 295: 676-680.

41. Higgins JP, Deeks JJ (2011) Chapter 7: selecting studies and collecting data. In: Higgins JP, Green S, ed. Cochrane handbook for systematic reviews of interventions (Version 5.1.0). Available on http://www.cochrane-handbook. org/.

42. Maeda K, Tsutamoto T, Wada A, Hisanaga T, Kinoshita M (1998) Plasma brain natriuretic peptide as a biochemical marker of high left ventricular enddiastolic pressure in patients with symptomatic left ventricular dysfunction. Am Heart J 135: 825-832.

43. Prastaro M, Paolillo S, Savarese G, Dellegrottaglie S, Scala O, et al. (2011) Nterminal pro-b-type natriuretic peptide and left atrial function in patients with congestive heart failure and severely reduced ejection fraction. Eur J Echocardiogr 12: 506-513.

44. Metra M, Zacà V, Parati G, Agostoni P, Bonadies M, et al. (2011) Cardiovascular and noncardiovascular comorbidities in patients with chronic heart failure. J Cardiovasc Med 12: 76-84.

45. Hildebrandt P, Collinson PO, Doughty RN, Fuat A, Gaze DC, et al. (2010) Agedependent values of $\mathrm{N}$-terminal pro-B-type natriuretic peptide are superior to a single cut-point for ruling out suspected systolic dysfunction in primary care. Eur Heart J 31: 1881-1889.

46. Steiner J, Guglin M (2008) BNP or NTproBNP? A clinician's perspective. Int J Cardiol 129: 5-14. 